\title{
Estudo sobre o crescimento e desenvolvimento craniofacial: teste de associação entre marcado- res genéticos e indicadores morfológicos numa amostra de fissurados labiopalatais do estado do Paraná - Brasil
}

\author{
Alcion Alves Silva*
}

Resumo

Objetivo: as fissuras que acometem a região do lábio e/ou palato são as anomalias faciais mais freqüentes nos seres humanos. Sua etiologia é complexa, envolvendo fatores genéticos e ambientais. Nos serviços multidisciplinares, a investigação científica das características desta deformidade sobre a população assistida é fundamental para a atuação em nível preventivo. A proposição deste estudo foi avaliar a possibilidade da estimativa do risco de fissuras labiopalatais, numa amostra de indivíduos afetados do estado do Paraná, através do teste de associação entre indicadores morfológicos (fenótipo) e uma região genética principal (genótipo). Metodologia: utilizou-se o complexo do Antígeno Leucocitário Humano (HLA) como marcador. Uma amostra composta por 17 indivíduos, do gênero masculino, acometidos por fissuras labiopalatais, não sindrômicos, oriundos do Centro de Atendimento Integral ao Fissurado (Curitiba/PR), foi testada quanto à associação entre duas características morfológicas da base do crânio - ângulo basicraniano (Ba.S.Na) e dimensão da base craniana anterior (BCA) - e os tipos HLA mais freqüentes. Resultados e Conclusões: o coeficiente de associação demonstrou-se fraco entre as grandezas cefalométricas representativas da morfologia basicraniana (Ba.S.Na e BCA). Entre a grandeza Ba.S.Na e os aloantígenos HLA-B, HLA-DQ e a grandeza BCA e os aloantígenos HLA-A, HLA-B, HLA-DQ e HLA-DR o coeficiente de associação indicou independência. Entre Ba.S.Na e os aloantígenos HLA-A e HLA-DR o coeficiente de associação indicou dissociação fraca. Como conclusão, não foi observada associação entre as características fenotípicas e genotípicas testadas para esta amostra, embora não tenha sido lícito afirmar ou negar a possibilidade de utilização destas como indicadores de risco para fissuras labiopalatais em outras populações.

Palavras-chave: Crescimento e desenvolvimento craniofacial. Fissuras labiopalatais. Genética.

* Mestre em Ortodontia. Doutor em Odontologia - Universidade Federal do Rio de Janeiro (UFRJ). 


\section{INTRODUÇÃO}

Embora as fissuras lábiais e/ou palatais (FL/P) sejam consideradas um problema de saúde pública no Brasil, a adoção de medidas preventivas é dificultada pela característica multifatorial da deformidade. A forma mais freqüente para estimar o risco de recorrência é a estimativa de risco empírico, porém por ser esta uma média da população, é uma determinação inexata para famílias individuais. A identificação de características morfológicas e/ou genéticas nos indivíduos afetados pode possibilitar a estimativa de risco de recorrência desta anomalia de forma mais precisa. Esta premissa é suportada pela literatura científica através de duas hipóteses:

1) Hipótese do modelo multifatorial ${ }^{10}$ - descreve a suscetibilidade da deformidade numa curva de distribuição contínua, com um limite além do qual os indivíduos manifestarão o defeito (Fig. 1). Esta hipótese aceita que a expressão de algum atributo morfológico da forma facial possa estar relacionada ao grau de risco para a patologia, sendo que a presença de características morfológicas próprias de indivíduos afetados por FL/P é sustentada pela literatura ${ }^{11,15,23}$.

2) Hipótese do gene principal - sustenta a existência de um fator genético primário, associado à etiologia das FL/P, sendo o braço curto do cromossomo 6 (6p21.31) uma das regiões prováveis ${ }^{16}$. Esta região (Fig. 2) abriga o Complexo Principal de Histocompatibilidade (MHC), um grupo de mais de

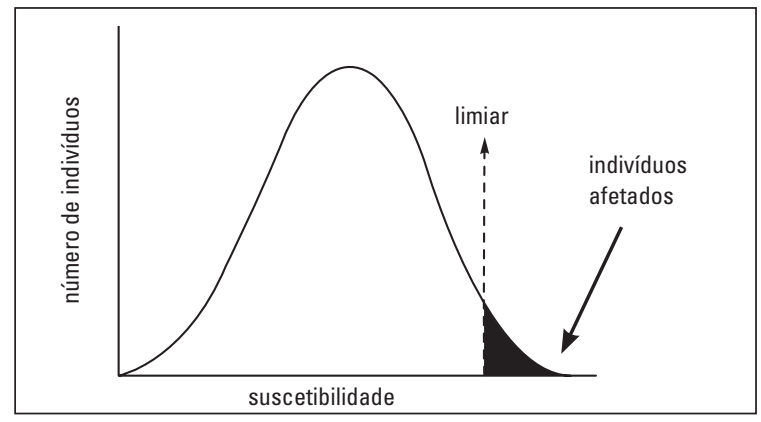

FIGURA 1 - Representação do modelo do limiar (baseado em THOMPSON, McINNES \& WILLARD, 1993).
224 genes designados em humanos por uma variante chamada Antígeno Leucocitário Humano (HLA) ${ }^{2,18}$. A maior função das moléculas codificadas pelo HLA é permitir o reconhecimento por efetores do sistema imunológico ${ }^{19}$, além de sua relação com a etiologia de mais de 40 patologias.

A avaliação simultânea das duas hipóteses pode ser procedida através do delineamento de um plano fatorial de investigação, o qual possibilita o teste de múltiplas variáveis.

Apesar da determinação precisa do comportamento dos genes relacionados à etiologia das FL/P não ser clara, devido a diversos fatores - diferentes genes que podem estar ativos em diferentes famílias $^{9}$, modificação da expressão genética em diferentes tipos raciais ${ }^{8,13}$ e ocorrência de expressão variável frente aos fatores ambientais ${ }^{25}$ - vários genes candidatos à associação com a deformidade em questão têm sido identificados e estudados, principalmente através de estudos de ligação em humanos e modelos animais. Entre os candidatos estão: TGFA (transforming growth factor alpha) e RARA (retinoic acid receptor alpha), 13A e o gene END1 ${ }^{4,6}$.

A proposição deste estudo foi avaliar a possibilidade da estimativa de risco para fissuras labiopalatais em famílias particulares, através do teste de associação entre indicadores morfológicos (fenótipo) e uma região genética principal (genótipo), utilizando o complexo HLA como marcador.

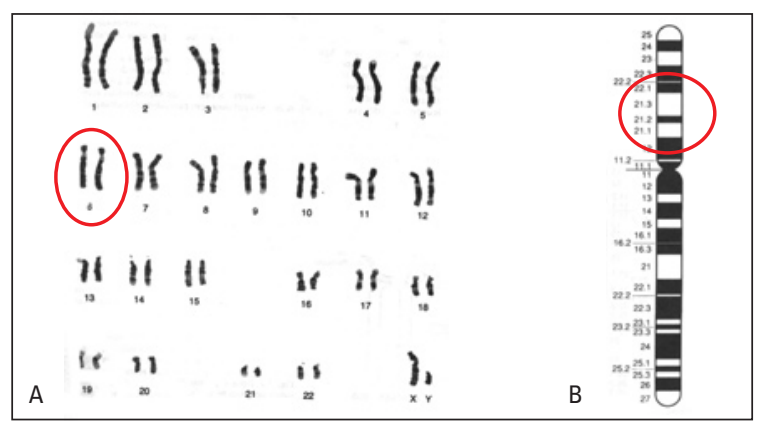

FIGURA 2 - A) 0 cariótipo humano (masculino), com o cromossomo número 6 em destaque. B) Região na qual se localiza o complexo maior de histocompatibilidade no cromossomo 6 . 


\begin{tabular}{|c|c|c|}
\hline $\begin{array}{l}\text { relação de associação entre } \\
\text { atributos }\end{array}$ & $\begin{array}{l}\text { coeficiente de } \\
\text { associação }\end{array}$ & interpretação \\
\hline Ba.S.Na x BCA & $+0,333$ & $\begin{array}{l}\text { associação } \\
\text { fraca }\end{array}$ \\
\hline Ba.S.Na x HLA-A & $-0,625$ & $\begin{array}{l}\text { dissociação } \\
\text { fraca }\end{array}$ \\
\hline BCA $\times$ HLA-A & 0 & independência \\
\hline Ba.S.Na x HLA-B & 0 & independência \\
\hline BCA $\times$ HLA-B & 0 & independência \\
\hline Ba.S.Na x HLA-DO & 0 & independência \\
\hline BCA $\times$ HLA-DO & 0 & independência \\
\hline Ba.S.Na $x$ HLA-DR & $-0,063$ & $\begin{array}{l}\text { dissociação } \\
\text { fraca }\end{array}$ \\
\hline BCA $x$ HLA-DR & 0 & independência \\
\hline
\end{tabular}

Quadro 1 - Interpretação dos resultados do coeficiente de associação.

\section{MATERIAL E MÉTODOS}

\section{Seleção da amostra}

A amostra foi composta por 17 indivíduos masculinos, acometidos por fissuras pré ou transforâme, não sindrômicos, oriundos do Centro de Atendimento Integral ao Fissurado, localizado na cidade de Curitiba, estado do Paraná. Todos os dados coletados foram resultado de exames requisitados no protocolo de tratamento dos pacientes, não sendo exclusivos para utilização científica, respeitando a Resolução 196/96 e a declaração de Helsinky.

\section{Técnicas para a coleta de dados Cefalometria}

A região craniofacial selecionada para avaliação das características morfológicas foi a base do crânio, em norma lateral (Fig. 3), por sua determinação genética durante o crescimento e desenvolvimento ${ }^{20}$, por apresentar crescimento precoce em relação ao complexo facial e por sua íntima relação com as deformidades dentofaciais ${ }^{1}$.

Utilizando telerradiografias em norma lateral da amostragem selecionada, procedeu-se o desenho anatômico da base do crânio e, sobre este, a técnica cefalométrica segundo descrição de Sil$\mathrm{va}^{22}$, a qual possibilitou avaliar as grandezas descritas a seguir (Fig. 4).

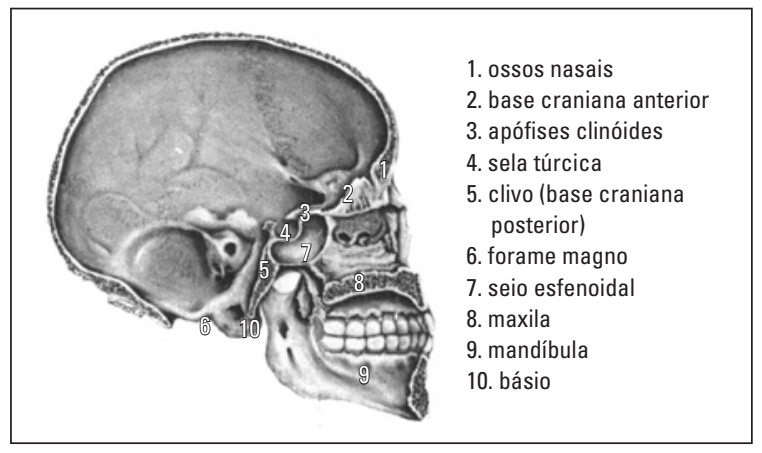

FIGURA 3 - Corte da cabeça no plano sagital mediano, ilustrando a base do crânio e sua relação com o complexo facial (baseado em ENLOW, 1982).

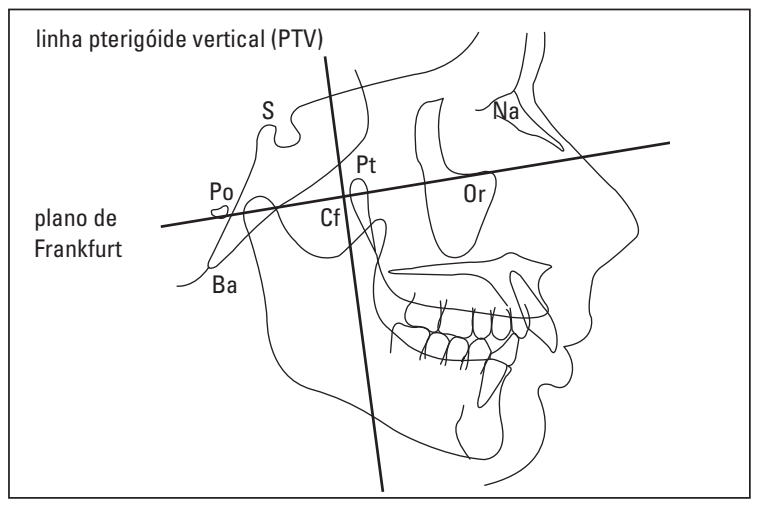

FIGURA 4 - Desenho anatômico e cefalograma indicando os pontos Sela (S), Násio (Na), Pório (Po), Centro da Face (CF), Pterigóide Vertical (Pt), Orbital (Or), Básio (Ba), além da Linha Pterigóide Vertical (PTV) e do Plano de Frankfurt.

a) Ângulo básio-sela-násio (Ba.S.Na) - grandeza angular formada pela interseção das linhas básio/sela e sela/násio, representa a deflexão da base do crânio;

b) Base craniana anterior (BCA) - grandeza relativa que representa a base craniana anterior, obtida através da equação (Na-PTV x 100 / Ba-Na); sendo estatisticamente, um dado derivado, representado percentualmente.

\section{Tipagem HLA}

Para as tipagens do complexo HLA de Classe I e II, da amostra experimental, foram coletados $20 \mathrm{ml}$ de sangue periférico, em tubo Vacutainer contendo heparina sódica, para isolamento dos linfócitos pela técnica de Böyum ${ }^{3}$. Um ul da suspensão de células T e B foi colocado nas cavidades 
de placas One-lambda special monoclonal typing tray set, constituído por 144 soros para Classe I (placas SMT144A e SMT144B) e 72 soros para Classe II (placas SMDR72), incluindo soros de controle positivo e negativo.

\section{Interpretação}

Escore 1 - negativo: proporção de células lisadas de $0 \%$ a $10 \%$;

Escore 2 - negativo duvidoso: proporção de células lisadas de $11 \%$ a $20 \%$;

Escore 4 - positivo duvidoso: proporção de células lisadas de $21 \%$ a $50 \%$;

Escore 6 - positivo: proporção de células lisadas de $51 \%$ a $90 \%$;

Escore 8 - fortemente positivo: proporção de células lisadas de $91 \%$ a $100 \%$.

\section{Metodologia estatística e análise dos dados}

O delineamento estatístico considerou a distribuição de freqüência simples (absoluta e relativa) dos argumentos Ba.S.Na, BCA, HLA-A, HLA-B, HLA-DQ, HLA-DR, para determinação da média

Tabela 1 - Resultados da análise cefalométrica.

\begin{tabular}{ccc}
\hline amostra & \multicolumn{2}{c}{ grandezas } \\
\hline identificação & Ba.S.Na & BCA \\
\hline 879 & $127^{\circ}$ & $56,1 \%$ \\
1055 & $138^{\circ}$ & $57,4 \%$ \\
2165 & $132^{\circ}$ & $52,6 \%$ \\
785 & $123^{\circ}$ & $59,4 \%$ \\
1712 & $123,5^{\circ}$ & $59,8 \%$ \\
289 & $123^{\circ}$ & $58,6 \%$ \\
1683 & $123,5^{\circ}$ & $55,4 \%$ \\
2069 & $125^{\circ}$ & $53,9 \%$ \\
2111 & $136^{\circ}$ & $53,4 \%$ \\
2125 & $128^{\circ}$ & $53,8 \%$ \\
2162 & $125^{\circ}$ & $58,1 \%$ \\
468 & $127^{\circ}$ & $56,7 \%$ \\
434 & $123^{\circ}$ & $52,1 \%$ \\
2430 & $128,5^{\circ}$ & $52,6 \%$ \\
2208 & $121^{\circ}$ & $54,0 \%$ \\
373 & $130^{\circ}$ & $57,5 \%$ \\
1323 & $124^{\circ}$ & $53,4 \%$ \\
\hline
\end{tabular}

aritmética (A), o cálculo do desvio padrão (s) para revelar a dispersão do conjunto em torno da média, o estabelecimento de um critério representativo das características fenotípicas e genotípicas mais significativas para os atributos considerados. Para tal, os indivíduos dentro da média aritmética e dos campos do desvio-padrão, representado em duplo sinal, foram considerados como portadores do atributo característico observado em indivíduos afetados, os demais foram considerados não portadores do atributo. A tabulação dos dados para o teste de associação ou independência dos atributos encontra-se abaixo:

\begin{tabular}{|c|c|c|c|}
\hline & \multicolumn{3}{|c|}{ atributo $Y$} \\
\hline atributo X & $\operatorname{sim}(B)$ & não (B) & total \\
\hline $\operatorname{sim}(A)$ & (A.B) & (A.B) & $(A)$ \\
\hline não $(\alpha)$ & $(\alpha . B)$ & $(\alpha . B)$ & $(\alpha)$ \\
\hline total & (B) & (b) & $N$ \\
\hline
\end{tabular}

A tabulação dos dados permitiu a aplicação da fórmula do Coeficiente de Associação (CA):

$$
C A=\frac{(A B) \cdot(\alpha \beta)-(A b) \cdot(\alpha B)}{(A B) \cdot(\alpha \beta)+(A b) \cdot(\alpha B)}
$$

A interpretação dos resultados considerou a seguinte aplicação dos critérios para determinação do relacionamento ou independência entre os atributos:

$\mathrm{CA}=-1$ = dissociação completa;

$\mathrm{CA}=0$ = independência;

$\mathrm{CA}=+1=$ associação completa.

\section{RESULTADOS}

Os resultados podem ser vistos nas tabelas 1 e 2 e no quadro 1 .

\section{DISCUSSÃO}

Sobre os limites inerentes ao delineamento adotado

O delineamento de pesquisa experimental objetivou testar o coeficiente de associação entre duas supostas características presentes nos indivíduos acometidos pela deformidade em estudo.

Em relação à precisão dos instrumentos utili- 
zados para coleta dos dados, as diferenças morfométricas obtidas através de análises cefalométricas são dados derivados de instrumento pouco refinado para inferências, assim devem ser avaliadas com critério para identificar se, apesar de presentes, são significantes em relação à variação observada na população normal. Ainda em relação às características morfométricas, levanta-se a questão que as mesmas podem ser um fator associado em determinada

Tabela 2 - Resultados da tipagem do antígeno leucocitário humano (HLA).

\begin{tabular}{ccccc}
\hline ID* & HLA-A & HLA-B & HLA-D0 & HLA-DR \\
\hline 879 & $24(9), 11$ & $44(12), 61(40)$ & $7(3), 8(3)$ & 4 \\
1055 & 2 & $38(16)$ & $8(3), 4$ & 4,8 \\
2165 & $2,29(19)$ & $44(12), 16$ & 2,4 & 7,8 \\
785 & 2,3 & $44(12), 57(17)$ & $7(3), 9(3)$ & $7,11(5)$ \\
1712 & $1,68(28)$ & $49(21), 35$ & $6(1)$ & $11(5), 15(2)$ \\
289 & $24(9), 31(19)$ & $35,75(15)$ & $2,7(3)$ & $7,11(5)$ \\
1683 & $29(19), 31(19)$ & $35,51(5)$ & 2,4 & 7,8 \\
2069 & $2,24(9)$ & $51(5), 35$ & $8(3), 4$ & 4,8 \\
2111 & $3,33(19)$ & $44(12), 64(15)$ & $6(1)$ & $1,13(6)$ \\
2125 & $2,29(19)$ & $45(12), 62(15)$ & $6(1), 7(3)$ & $14(6)$ \\
2162 & $2,33(19)$ & $65(14), 60(40)$ & $5(1)$ & $1,13(6)$ \\
469 & 1,2 & 13,18 & $2,7(3)$ & $7,11(5)$ \\
434 & $2,32(19)$ & $51(5), 44(12)$ & $7(3)$ & $4,14(6)$ \\
2430 & $1,34(10)$ & $44(12), 18$ & $2,6(1)$ & $7,15(2)$ \\
2208 & $2,68(28)$ & 27,35 & $5(1), 4$ & 1,8 \\
1323 & $2,31(19)$ & $39(16), 71(70)$ & $6(1), 4$ & $8,13(6)$ \\
373 & $23(9), 2$ & $49(21), 55(22)$ & $5(1), 2$ & $1,11(5)$ \\
\hline
\end{tabular}

*Número de identificação do prontuário nos arquivos do Centro de Atendimento ao Fissurado - Curitiba/Pr.

\begin{tabular}{ccc}
\hline $\begin{array}{c}\text { relação de associação } \\
\text { entre atributos }\end{array}$ & $\begin{array}{c}\text { coeficiente de } \\
\text { associação }\end{array}$ & interpretação \\
\hline Ba.S.Na $\times$ BCA & $+0,333$ & associação fraca \\
Ba.S.Na $\times$ HLA-A & $-0,625$ & dissociação fraca \\
BCA $\times$ HLA-A & 0 & independência \\
Ba.S.Na $\times$ HLA-B & 0 & independência \\
BCA $\times$ HLA-B & 0 & independência \\
Ba.S.Na $\times$ HLA-DO & 0 & independência \\
BCA $\times$ HLA-DO & 0 & independência \\
Ba.S.Na $\times$ HLA-DR & $-0,063$ & dissociação fraca \\
BCA $\times$ HLA-DR & 0 & independência \\
\hline
\end{tabular}

Quadro 1 - Interpretação dos resultados do coeficiente de associação. população, porém esta manifestação pode não ser consistente para outro grupo da mesma população exposto a diferentes fatores ambientais. Considerase ainda que a hipótese das características craniofaciais é limitada para explicar a relação causa-efeito, desta forma, não foi intenção deste estudo este nível de inferência, mas sim a confirmação ou negação de associação entre duas características observadas na amostra estudada. Quanto à precisão esperada para o instrumento de coleta dos dados genotípicos, a técnica bioquímica empregada, descrita como tipagem HLA, representa uma forma indireta de determinação da expressão genética, através da detecção dos produtos genéticos, não representando imprecisão sobre os dados levantados.

\section{Sobre os resultados obtidos pelo teste de as- sociação entre indicadores genéticos e mor- fológicos}

Os resultados do teste de associação entre as características fenotípicas da base do crânio (Ba.S.Na e BCA) e genotípicas (utilizando o complexo HLA como marcador), para a amostra selecionada no estudo piloto, composta por 17 indivíduos do gênero masculino, todos afetados por fissuras envolvendo lábio e/ou palato, demonstraram os níveis de associação descritos a seguir.

Testado preliminarmente o coeficiente de associação (CA) entre as duas características morfológicas selecionadas, o ângulo basicraniano (Ba.S.Na) e a base craniana anterior (BCA), os resultados demonstraram associação fraca $(\mathrm{CA}=+0,333)$. O resultado concorda com a hipótese proposta por Enlow ${ }^{7}$, sobre os equivalentes de crescimento, segundo a qual o crescimento balanceado é resultado da equivalência do crescimento de partes específicas e suas contrapartes, desta forma, a associação fraca é o que provavelmente se poderia esperar de duas regiões que não são equivalentes de crescimento direto, porém nas quais o processo de crescimento regular mantém a equivalência entre as partes.

Os resultados do coeficiente de associação en- 
tre os indicadores morfológicos e genéticos indicaram independência $(C A=0)$ entre as grandezas BCA e os aloantígenos HLA-A, HLA-B (Classe I), HLA-DQ e HLA-DR (Classe II), e Ba.S.Na. e os aloantígenos HLA-B e HLA-DQ, negando a hipótese de trabalho que considerava a probabilidade de existência de associação entre as características fenotípicas e genotípicas testadas. Embora a morfologia craniofacial tenha sido relatada na literatura com particularidades próprias para os indivíduos acometidos pela deformidade em questão, bem como para seus familiares ${ }^{12,17,27}$, e a possibilidade destas serem utilizadas como indicadores de risco, o mesmo ocorrendo com o HLA quanto à sua relação com a etiologia das fissuras labiais e/ou palatais ${ }^{4}$, o que os resultados deste estudo evidenciaram foi a ausência de associação entre os dois fatores, não discordando dos autores supracitados quanto à presença de características morfológicas e genéticas específicas nos indivíduos acometidos pela deformidade.

Apesar da hipótese de trabalho, baseada nos estudos de Hermann et al. ${ }^{12}$, ter aceito que as assimetrias faciais na dimensão ântero-posterior podem apresentar maior herdabilidade por sua etiologia estar relacionada a genes reguladores durante a embriogênese, e por este motivo esta investigação considerou a avaliação de telerradiografias em norma lateral (ântero-posterior), há de se observar que as fissuras labiopalatais são deformidades primariamente relacionadas à dimensão transversal, apesar de vários trabalhos apresentarem esta dismorfologia como polimórfica ${ }^{14,24}$, afetando inúmeras regiões de organismo. A inferência sobre as proposições construídas possibilita direcionar novos estudos para confirmar estes resultados ou testar a associação de outros indicadores morfológicos e genéticos, variando suas características.

$\mathrm{Na}$ avaliação dos resultados, as associações entre as grandezas Ba.S.Na e HLA-A, bem como Ba.S.Na. e HLA-DR apresentaram coeficiente de associação negativo, respectivamente $\mathrm{CA}=-0,625 \mathrm{e}$ $\mathrm{CA}=-0,063$, indicando dissociação fraca entre estas.
Sobre a possibilidade de estimar o risco de recorrência das fissuras labiais e/ou palatais através dos indicadores testados

Quanto à utilização dos marcadores morfológicos e genéticos investigados, estes não apresentaram um grau de associação (CA) que os credenciasse para a estimativa de risco aumentado das fissuras labiais e/ou palatais, porém os resultados não se relacionam à relação individual de cada fator com a deformidade, conforme vários estudos suportaram ${ }^{12,17}$, além do que, a incógnita supradiscutida permanece tentadora para novos estudos. Seria o fraco coeficiente de associação observado resultado da própria dismorfologia congênita?

De acordo com as características de simplicidade ou complexidade, definida pelo número de variáveis que intervém no problema e das características de definição ou indefinição, pela precisão das relações que ligam as variáveis, o fenômeno estudado caracterizou-se como complexo-indefinido. Isto quer dizer que inúmeras variáveis podem atuar simultaneamente para a ocorrência das fissuras labiais e/ou palatais, variando ainda a forma com que estas se relacionam para produção da deformidade. Assim sendo, outras características morfológicas e genéticas podem apresentar algum grau de associação para as fissuras, ou mesmo as características estudadas podem apresentar significância na associação em diferentes modos de relação.

A identificação de genes relacionados às deformidades craniofaciais é apenas uma fase para o entendimento dos mecanismos de crescimento e desenvolvimento normal ou anormal, sendo que a ênfase no desenvolvimento de novas técnicas e tecnologias biomoleculares para investigação desta relação, com aplicação prática na melhoria da qualidade de vida dos pacientes constitui tendência para as próximas décadas ${ }^{28}$.

\section{CONCLUSÃO}

Embora o teste de associação entre os indicado- 
res fenotípicos e marcadores genotípicos tenha indicado independência entre estes, não foi lícito afirmar ou negar a possibilidade da utilização dos mesmos como indicadores de risco aumentado para as fissuras labiais e/ou palatais na amostra estudada, visto a relação de complexidade e indefinição das variáveis envolvidas, requerendo o teste de novas hipóteses.

\title{
Craniofacial growth and development study through the association between genetic markers and morphological indicators at cleft lip and palate sample in Paraná state - Brazil
}

\begin{abstract}
Aim: the cleft lip and palate are the most frequent human face deformities. It has a complex etiology with genetic and environmental factors. At the multidisciplinary services, a scientific research about deformities features is important in order to act at preventive levels. The purpose of this study was to evaluate the recurring risk of cleft lip and palate, among the affected population of the state of Paraná (Brazil), through morphologic and genetic indicators. Methods: a sample of 17 cleft lip and palate affected individuals, not syndromic, males, selected from Center for Cleft Lip and Palate Assistance in Curitiba (PR), was tested to association between 2 morphologic features of cranial base - cranial base angle (Ba.S. Na) and anterior cranial base dimension (BCA) - and more frequent HLA types. The association coefficient between cranial base morphologic values (Ba.S.Na and BCA) proved to be low. Results and Conclusions: the association coefficient test for the values between Ba.S.Na and aloantigens HLA-B, HLADQ; BCA and aloantigens HLA-A, HLA-B, HLA-DQ and HLA-DR proved independence. The coefficient between Ba.S.Na and aloatigens HLA-A and HLA-DR showed weak dissociation. The outcome showed no association between phenotypic and genotypic characteristics tested at this sample, although wasn't possible to confirm or not the possibility of utilization of them like risk indicators to cleft lip and/or palate at another population.
\end{abstract}

Key words: Craniofacial growth and development. Cleft lip and palate. Genetics.

\section{REFERÊNCIAS}

1. ALOISIO, D. D.; KULBERSH, V. P. A comparative and correlational study of the cranial base in North American blacks. Am J Orthod Dentofacial Orthop, St. Louis, v. 102, no. 5, p. 449-455, 1992.

2. BECK, S.; GERAGHTY, D.; INOKO, H.; ROWEN, L. Complete sequence and gene map of a human major histocompatibility complex. Nature, London, v. 401, p. 921-923, 1999.

3. BÖYUM, A. Isolation of mononuclear cells and granulocytes from blood. Scand J Clin Lab Invest, London, v. 21, n. 97, p. $77-89,1968$

4. CARINCI, F.; PEZZETI, F.; SCAPOLI, E. P.; BACILIERO, U.; CURIONI, C.; TOGNON, M. Nonsyndromic cleft lip and palate: evidence of linkage to a microsatellite marker on 6p23. Am J Hum Genet, Chicago, v. 56, p. 337-339, 1995

5. CHENEVIX-TRENCH, G.; JONES, K.; GREENE, A. C.; MARTIN, N. G. Further evidence for an association between genetic variation in transforming growth factor alpha and cleft lip palate. Am J Hum Genet, Chicago, v. 48, p. 1012-1013, 1991.

6. CHENEVIX-TRENCH, G.; JONES, K. GREENE, A. C. DIFFY, D. L.; MARTIN, N. G. Cleft lip with or without cleft palate: association with transforming growth factor alpha and retinoic loci. Am J Hum Genet, Chicago, v. 51, p. 1377-1385, 1992.
7. ENLOW, D. H. Interviews: Dr. Donald H. Enlow. J Clin Orthod Boulder, p. 669-679, Oct. 1982

8. FANG, H.; SASSANI, R.; BARTELETT, S. P.; LEE, A.; HECHT, J. T.; MALCOM, S.; WINTER, R. M. Evidence from family studies for linkage disequilibrium between TGFA and gene for nonsyndromic cleft lip with or without cleft palate. Am J Hum Genet, Chicago, v. 55, p. 932-936, 1994.

9. FARRAL, M.; HOLDER, S. Familial recurrence-pattern analysis of cleft lip with or without cleft palate. Am J Hum Genet, Chicago, v. 50, no. 2, p. 270-277, 1992.

10. FRASER, F. C. Some under looked properties of the multifactorial/ threshold model. Am J Hum Genet, Chicago, v. 62, p. 1262-1265, 1998.

11. FRIEDE, H. Growth sites and growth mechanisms at risk in cleft lip and palate. Acta Odontol Scand, Oslo, v. 56, no. 6, p. 346351,1998

12. HERMANN, N. V.; JENSEN, B. L.: DAHL, E.; BOLUND, S KREIBORG, S. Craniofacial comparsions in 22-month old lip operated children with unilateral complete cleft lip and palate and unilateral incomplete cleft lip. Cleft Palate Craniofac J, Lewiston, v. 37, no. 3, p. 303-317, 2000.

13. JARRA, L.; BRANCO, R.; CHIFFELLE, I.; PALOMINO, H.; CARREÑO, $H$. Evidence for an association between RFLPs at the transforming growth factor alpha (locus) and nonsyndromic cleft lip/palate in a South American population. Am J Hum Genet Chicago, v. 56, p. 339-341, 1995. 
14. MISHIMA, K: MORI, Y: SUGAHARA, T: SAKUDA, M. Comparsion between the palatal configurations in complete and incomplete unilateral cleft lip and palate infants under 18 months of age. Cleft Palate Craniofac J, Lewiston, v. 38, no. 1, p. 49-54, 2001.

15. MOSSEY, P. A.; McCOLL, J.; O'HARA, M. Cephalometric features in the parents of children with orofacial clefting. Br J Oral Maxillofacial Surg, v. 36, n. 3, p. 202-12, 1998.

16. MURRAY, J. C. Face facts: genes, environment and clefts. Am J Hum Genet, Chicago, v. 57, p. 227-232, 1995.

17. NOPOULOS, P.; BERG, S.; CANADY, J.; RICHMAN, L.; DEMARK, D. V.; ANDREASEN, N. C. Abnormal brain morphology in patients with isolated cleft lip, cleft palate or both: a preliminary analysis. Cleft Palate Craniofac J, Lewiston, v. 37, no. 5, p. 441-446, 2000

18. PARHAM, P. O sistema imune. Porto Alegre: Artmed, 1999.

19. PAUL, W. E. Fundamental immunology. Philadelphia: Lippincott-Raven, 1999.

20. PIRTTINIEMI, P. Normal and increased functional asymmetries in the craniofacial area. Acta Odontol Scand, Oslo, v. 56, no. 6, p. 342 345, 1998.

21. SEMB, G. A study of facial growth in patients with unilateral cleft lip and palate treated by the Oslo CLP team. The Cleft PalateCraniofacial Journal, [s.l.], v. 28, n. 1, p. 1-21, 1991.
22. SILVA, A. A. Previsão de crescimento: a relação entre a base do crânio e a classe III de Angle. R Dental Press Ortodon Ortop Maxilar, Maringá, v. 2, n. 4, p. 71-79, 1997.

23. SILVA FILHO, O. G.; MACHADO, F. M. C.; ANDRADE, A. C.; FREITAS, J. A. S.; BISHARA, S. Upper dental arch morphology of adult unoperated complete bilateral cleft lip and palate. Am J Orthod Dentofacial Orthop, St. Louis, v. 114, no. 2, p. 154161, 1998.

24. SPYROPOULOS, M. N.; BURDI, A. R. Patterns of body and viceral growth in human prenates with clefts of the lip and palate. Cleft Palate Craniofac J, Lewiston, v. 38, no. 4, p. 341-345, 2001

25. STEIN, J.; MULLIKEN, J. B.; STAL, S.; GASSER, D. L.; MALCOM, S.; WINTER, R.; BLANTON, S. H.; AMOS, C.; SEEMANOVA, E.; HECHT, J. T. Nonsyndromic cleft lip with or without cleft palate: evidence of linkage to BCL3 in 17 multigenerational families. Am J Hum Genet, Chicago, v. 57, p. 257-272, 1995.

26. THOMPSON, M. W.; McINNES, R. R.; WILLARD, H. F. Genética médica. 5. ed. Rio de Janeiro: Guanabara Koogan. 1993.

27. WILL, A. L. Growth and development in patients with untreated clefts. Cleft Palate Craniofac J, Lewiston, v. 34, no. 6, p. 523525, 2000.

28. WYSZYNSKI, D. F.; MITCHELL, L. E. Report of the newly formed international consortium for oral clefts genetics. Cleft Palate Craniofac J, Lewiston, v. 36, no. 2, p. 174-179, 1999.
Endereço de correspondência

Alcion Alves Silva

Rua Bruno Filgueira, 369/1502 - Batel

CEP: 80240-220 - Curitiba/PR

E-mail: cioccwb@hotmail.com 